\title{
Efectividad del método Enfoque Natural en la competencia léxica oral de estudiantes de inglés del instituto de idiomas de una universidad privada
}

\author{
Effectiveness of the Natural Approach method in the oral lexical competence of English \\ students from the Language Institute of a private university
}

CARI, Yaneth M. ${ }^{1}$

PAMPA, Noe B. ${ }^{2}$

\begin{abstract}
Resumen
La presente investigación tiene como objetivo evaluar la eficiencia del método Enfoque Natural en la competencia léxica de estudiantes del inglés. El muestreo de la investigación fue no probabilístico-intencional empleando el análisis de muestras pareadas. Los resultados en la competencias Pre fue 16.91 y Pos de 37.78, con un incremento de 20.87 (P-val < 0.05). Estos resultados permiten validar el método y la efectividad en la competencia léxica y sus dimensiones en complejidad, precisión y fluidez del inglés.

Palabras clave: método Enfoque Natural, competencia léxica oral, complejidad, exactitud y fluidez.

Abstract

The present research has the objective of evaluating the efficiency of the natural approach method in the lexical competence of English students. The research sampling was non-probabilisticintentional using paired sample analysis. The results in the Pre competencies were 16.91 and Pos 37.78 , with an increase of 20.87 ( $P$-val $<0.05$ ). These results allow to validate the method and the effectiveness in lexical competence and its dimensions in complexity, precision and fluency of English. key words: method natural approach, oral lexical competence, complexity, accuracy and fluency.
\end{abstract}

\section{Introducción}

Hoy en día se cataloga que el no aprender inglés podría representar una barrera que podría impedir el fortalecimiento de nuevos conocimientos (Choez, Stagg \& Morocho 2020). Y las posibles consecuencias de este problema incluyen una participación limitada de estudiantes en el desarrollo de las habilidades del habla y a consecuencia de ello un nivel bajo en la expresión oral, un nivel reducido de producción oral en la adquisición del idioma inglés y un desarrollo restringido de las actividades de producción oral que promueven la actividad personal y cultural de los estudiantes; sin embargo, para resolver los problemas respecto a mejorar las habilidades de comunicación del inglés, debe ser un foco importante conocer y aplicar la efectividad de los

\footnotetext{
${ }^{1}$ MSc in Education and Research, Teacher english language. Faculty of Human Sciences and Education. Universidad Peruana Unión. yaneth_c@upeu.edu.pe

2 PhD in Engineering Sciences, Teacher researcher in statistical methods. Faculty of Engineering. Universidad Nacional de Barranca. npampa@unab.edu.pe
} 
métodos, técnicas y estrategias para adquirir el idioma inglés. Guacho et al. (2019) indican que, de no adquirir el idioma inglés, en un futuro se registraría una limitada participación activa de los estudiantes para mejoramiento de actividades de expresión, un mínimo nivel de producción oral en la adquisición del idioma extranjero y un restringido desarrollo de producción oral que motive el interés personal y cultural de los estudiantes.

El uso del inglés como lengua extranjera hoy en día toma mayor fuerza, sobre todo cuando se da uso de manera oral, por eso existe una demanda no solamente nacional sino internacional, donde en casi todas las instituciones públicas, y privadas, sean de nivel básico regular o universitario, exigen dominar el inglés en un determinado nivel. Sin embargo, la carencia de conocimiento del léxico en general ha producido una gran limitación al momento de querer expresar un mensaje, lo que conlleva a que el mensaje no llegue de manera adecuada y la comunicación oral no sea efectiva en el entorno social donde éste se encuentre y muy pocas veces se trasmita el mensaje completo. La ineficiente competencia léxica conlleva a la ineficiencia de un idioma, ya que de un cierto nivel a otro puede llegar modificada, detenida, o filtrada según el nivel (Julluni \& Samata, 2019). Por ello que la adquisición de una segunda lengua es un proceso determinado por múltiples factores, y desarrollar la competencia léxica oral del inglés es esencial para la comprensión de una lengua extranjera (Jay Walker, 2009).

Jay Walker (2009) menciona que el idioma inglés se ha convertido en una manía mundial y explica que aproximadamente dos billones de personas intentan aprenderlo y hace una correlación que las matemáticas se consideran como el idioma de las ciencias, la música el de las emociones y el inglés el de la solución a los problemas internacionales hablando justamente de la economía, hambrunas y producción científica. Por otro lado Cervilla (2009) afirma que se ha reconocido que el inglés ha llegado a constituirse en un lenguaje global, es decir, una forma primaria de diálogo que ha permitido enlazar diferentes culturas.

Por otro lado, la competencia léxica oral se ha configurado como uno de los indicadores más precisos y representativos de las diferencias individuales halladas entre los estudiantes en lo que se refiere a su desarrollo lingüístico. En consecuencia el Ministerio de Educación (ME, 2020) en el Diseño Curricular Básico Nacional del Perú, señala que respecto al área de inglés tiene como referencia el Marco Común Europeo, porque integra todo lo que los estudiantes deben saber para comunicarse y actuar en distintos contextos.

A pesar del interés del Ministerio de Educación, solo solo 4 de cada 10 estudiantes universitarios peruanos tienen conocimiento acerca del inglés, de acuerdo a los resultados emitidos por el Instituto Nacional de Estadística e Informática (INEI). En una evaluación los resultados globales mejoraron (43.7\%), pero no se consideraron alentadores porque demostraron la brecha existente entre los estudiantes de la capital y las provincias: $55.7 \%$ de los estudiantes de Lima Metropolitana conocían el inglés como idioma, contra $30 \%$ en promedio en las provincias. Tal diferencia llevó a que en 2012 se aprobara el proyecto de Ley Na 2086/2012-CR, ley que estableció la obligatoriedad de la enseñanza del idioma inglés en los niveles de educación básica regular y nivel superior. Morán Flores et al. (2010) afirman que ese poco conocimiento del idioma fue adquirido durante la etapa de educación Básica Regular (Primaria y Secundaria).

A través de los años, los diferentes gobiernos peruanos han creado políticas de inclusión del inglés como materia de enseñanza y aprendizaje, pero no se ha seguido un plan de acción coherente y secuencial. Esto ha traido como consecuencia, entre otros aspectos, una escacez de profesores de inglés, que para 2015 era de 2300 y luego de 2000 anualmente, con el agravante de que $70 \%$ de quienes dictan la materia no son docentes especializados, tal y como lo indica el British Council (2015).

En septiembre de 2015 el entonces presidente Ollanta Humala aprobó la Política Nacional de Enseñanza, Aprendizaje y Uso del Idioma Inglés, con el nombre de "Inglés, puertas al mundo", con la finalidad de que los escolares desarrollen competencias comunicativas en el aprendizaje de mencionado idioma y puedan ampliar sus oportunidades de acceder a ofertas educativas, científicas, tecnológicas y laborales (British Council, 2015). 
Podría explicarse que, a pesar de los intentos gubernamentales por mejorar la enseñanza del idioma extranjero, persiste el problema de la enseñanza tradicional del lenguaje, tal y como lo explica British Council (2015). Es decir, se enseña a los estudiantes fundamentalmente la gramática por la gramática, sin tomar en cuenta la importancia que tiene la comunicación oral, ya que esta es necesaria al momento de comunicarse y entender el idioma en sí, tal y cual lo hace un niño de manera innata al momento de su crecimiento. A consecuencia de esto, los estudiantes desempeñan un papel pasivo, lo que hace que no aprendan el inglés ni les guste y si lo estudian lo hacen por presión o porque se considera un requisito como parte de la malla curricular o para obtener un certificado. Por ello, el perfeccionamiento de los metodos de enseñanza en inglés para la adquisición del nuevo idioma, debe plasmarse con métodos basados directamente en el desarrollo de la competencia oral, como Enfoque Natural, que propicia la solución de estos problemas a través del desarrollo de la competencia léxica oral, considerándose al docente como un facilitador y mediador.

A través del Enfoque Natural se busca la efectividad en la adquisición del vocabulario y sus procesos aplicativos. Asimismo esta metodología ayuda a lograr en los estudiantes la confianza, y romper barreras para que ellos ya no se sientan intimidados, con miedo o dificultad, sino confiados y sin temor a equivocarse. Asimismo, se persigue que los docentes se beneficien y puedan aplicar este y otros métodos, logrando así que los estudiantes del instituto de idiomas ya no sientan al aprendizaje del idioma como una obligación o un requisito para obtener algún grado académico, sino como parte de su formación profesional, adquiriendo una competencia que les abre puertas a oportunidades que ofrece el mundo de hoy.

Es asi que el presente trabajo de investigación tuvo como objetivo evaluar la eficiencia del método Enfoque Natural en la competencia léxica oral de estudiantes de inglés básico del Instituto de idiomas de una universidad privada.

\subsection{Método Enfoque Natural}

Este método fue desarrollado por Tracy. D Terrell's y Stephen Krashen (Krashen y Trerrell, 1983) quienes fundamentaron que todos los aprendices de cualquier edad son capaces de utilizar diferentes palabras al hablar. Esta teoría lleva a comprender la lengua extranjera a través de figuras, acciones y situaciones gramaticales y léxicas, ayudando a los estudiantes a usar la lengua extranjera no como una práctica artificial sino mediante la motivación del uso de la misma, comenzando con la utilización de palabras sueltas hasta llegar a un discurso coherente.

Asimismo, el Enfoque Natural tiene como objetivo hacer que el estudiante de nivel superior, primaria, seicuicidbaikance un nivel aceptable de comunicación. En general, el Enfoque Natural enfatiza la exposición a un input comprensible y significativo, más que a producciones gramaticales correctas. Puesto que el vocabulario es portador de significado, se considera que es importante en el proceso de adquisición de una lengua. El desarrollo del léxico se puede potenciar mediante la instrucción, que tiene que basarse en la explicación de la estructura del léxico (Morante, 2005).

Por ello Krashen y Terrel (1988) presentan al Enfoque Natural como un método efectivo para inducir a que todos los aprendices de cualquier edad sean capaces de adquirir diferentes palabras y luego emitirlas inconscientemente al hablar, y que la tarea del docente es hacer las clases interesantes y agradables donde cada uno pueda participar en actividades y juegos sin temor a equivocarse. Así los autores proponen conceptos entre la adquisición y aprendizaje donde claramente se percibe el contraste entre ambos.

Ramos (2003) menciona que el método Enfoque Natural introdujo dos innovaciones importantes frente a los métodos anteriores: separar a los estudiantes de acuerdo con sus niveles de inglés (preproduction, early production, speech emergente, intermediate) y tomar en cuenta sus necesidades específicas. 


\subsection{Competencia léxica oral}

Housen \& Kuiken (2009) mencionan que la capacidad para relacionar y formar palabras, frases con significados y utilizarlas adecuadamente se denomina competencia léxica, y al lugar donde reside el conocimiento de esa competencia se le denomina lexicón mental. Asimismo, Council of Europe (2001) define la competencia léxica como el conocimiento del vocabulario de una lengua y la capacidad para utilizarlo; posteriormente señala que la competencia semántica comprende la conciencia y el control de la organización del significado que posee el estudiante. Vivanco (2001) explica también por competencia léxica como el sentido del conocimiento que se debe poseer para poder utilizar la palabra con propiedad de la capacidad de reconocer, aprender, recuperar y relacionar las distintas palabras a nivel oral y escrito.

En los aspectos cualitativos del desarrollo léxico, Briseño (2013) menciona que la mayor parte del trabajo realizado en el área del vocabulario hasta los años ochenta se centra no en el aprendizaje del léxico, sino en la gestión del aprendizaje. Es decir, aprender palabras no es un acto acumulativo, basado en la memorización, sino que se trata de un proceso cognitivo complejo, que da lugar al desarrollo de un lexicón mental (Romero, 2018).

Por otro lado, el Oral Input, desarrollado por Krashen, indica que el estudiante debe estar sumergido en un entorno lingüístico favorable para que pueda adquirir una segunda lengua sin presión, para ello, desde la psicolingüística algunos autores abogan por un input comprensible, que vaya solo un poco más allá de los conocimientos del alumno y sea un factor sobresaliente (Slagter, 1994). Asimismo el autor plantea que la adquisición de una lengua es un proceso implícito en el que se interiorizan las reglas gramaticales cuando se está expuesto a textos orales o escritos, pero auténticos.

\subsection{Dimensión de complejidad, precisión, y fluidez}

Diversos estudios de investigación en lingüísticas aplicadas afirman que para la adquisición léxica de un segundo idioma, lengua extranjera o hablante nativo, existen tres dimensiones: complejidad, precisión y fluidez (CAF). Brumfit (1984) definió las actividades de fluidez y precisión. La complejidad, se añadió en la década de 1990, a raíz de Skehan (1989), quien propuso un modelo L2, que por primera vez incluye la CAF como las tres dimensiones principales de competencia.

La complejidad es encontrar una mayor precisión gramatical a expensas de la fluidez. Asimismo refleja la disposición del aprendiz para producir lengua en tiempo real sin hacer pausas injustificadas o dudar. Para ello se han utilizado diversas formas de medir la complejidad, como medir la velocidad del habla, la longitudinal de pausas, o la cantidad de falsos comienzos, de repeticiones o de reformulaciones (Skehan, 1996).

La precisión es lo que hace no equivocarse o pensar en pronunciar mal el inglés. Por eso al desarrollar el idioma inglés como segunda lengua, es necesario que se preocupe el estudiante en hacerlo mejor. Y si se preocupa por la precisión no se mejorará con respecto a la fluidez. Para conseguir hablar inglés se debe dar permiso para equivocarnos, pues solo así se puede llegar a la precisión (Mutluoglu y Ceylan, 2009).

La fluidez es de uso general en un sentido amplio, similar al dominio del segundo idioma (Koponen y Riggenbach, 2000). Para el autor el componente de la fluidez es especialmente propenso a ser evaluado de manera integral por los evaluadores, así como medidas de fluidez a base temporal. Y los evaluadores pueden ser especialmente vulnerables a responder a su construcción individual de la fluidez. El amplio sentido de la fluidez puede incluir las opciones léxicas, complejidad gramatical y pragmática. 


\section{Metodología}

El presente trabajo de investigación corresponde a un enfoque de investigación cuantitativa, diseño pre experimental, con pre y post prueba, aplicadas a un grupo de estudio. La población fue de 254 estudiantes y la muestra estuvo conformada por 23 estudiantes del básico II de un Instituto de Idiomas de la Universidad Peruana Unión, Perú.

El proceso de muestreo de la investigación fue no probabilístico-intencional y para conducir éste estudio respecto a sus variables, dimensiones y de sus procedimientos y operación de variables, se tomó en cuenta la hipótesis si el método Enfoque Natural es eficiente en las dimensiones de complejidad, exactitud, fluidez y complejidad del léxico oral.

Para la presente investigación, el método en estudio se realizó a través de sesiones de aprendizaje basados en lessons plans apoyado por el Departamento de idiomas de la Universidad de California, San Diego (Terrell, 1985), con una duración de 10 sesiones adaptadas desde el pretest hasta el postest.

\subsection{Instrumento de observación}

El instrumento ficha de observación fue adaptado según la metodología del Department of Spanish and Portuguese Studies (2012). El mencionado instrumento estuvo dividido en tres partes, una por dimensión. Las variables de estudios fueron las dimensiones de complejidad, exactitud y fluidez, cada uno con cuatro indicadores que ayudaron a medir la competencia léxica oral del inglés.

Fueron consideradas cuatro escalas de valoración: Alto, Regular, Bajo e Insatisfactorio. Y para las tres dimensiones se consideraron las escalas vigesimales de 13 a 16 (Alto), de 10 a 12 (Regular), de 7 a 9 (Bajo) y de 4 a 6 (Insatisfactorio). Para la variable Competencia Léxica Oral se tomó en cuenta la escala de valoración de 39 a 4 (Alto), de 30 a 38 (Regular), de 21 a 29 (Bajo) y de 12 a 20 (Insatisfactorio).

El instrumento fue validado por expertos donde evaluaron la claridad, congruencia, contexto y el dominio del constructo afirmándose de esa manera que hay una buena relación entre los ítems indicados. Luego, se procedió a la aplicación del instrumento antes y después del método. Para el desarollo del método se emplearon diez sesiones de clase empleando el modelo y adaptación del departamento de idiomas de la universidad de California San Diego (Terrell, 1985).

\subsection{Diseño y análisis estadístico}

Con la finalidad de comprobar la fiabilidad y validez del instrumento utilizado, previo a la recolección de datos, el instrumento fue aplicado a una prueba de campo y los datos obtenidos se sometieron a la prueba Alpha de Cronbach.

$$
T_{k k}=\frac{K[\operatorname{media}(r)]}{1+[(k-1)(\operatorname{media}(r))]}
$$

Donde:

"k": Es el número de reactivos de la prueba

Media(r): Es la medida de las correlaciones entre los reactivos

Tkk: Es el coeficiente de confiabilidad

Para verificar la influencia de la metodología del Enfoque Natural en las dimensiones y competencias antes y después de la aplicación, se empleó la prueba t para muestras pareadas para comparar las medias de dos series de mediciones realizadas sobre las mismas unidades estadísticas y la correlación de person para verificar las correlaciones entre los las dimensiones y competencia. 
Para el análisis estadístico se aplicó la distribución normal con Chapiro Wilk y correlación de Pearson y los datos se analizaron mediante la prueba t de Student de muestras relacionadas a un intervalo de confianza de $95 \%$ $(\propto<0.05)$. Por otro lado, el tratamiento y análisis estadístico de los datos obtenidos se procesó con el programa estadístico Minitab, versión 19.1

\section{Resultados}

La Tabla 1 muestra los resultados de los coeficientes de Alpha de Crombach para las dimensiones de complejidad, precisión y fluidez. Los valores se sitúan entre 0.67 a 0.79 , indicando una buena fiabilidad y validez del instrumento utilizado en relación a los ítems señalados en la variable del desarrollo de las dimensiones. Con relación a la competencia de lexical oral, muestra un coeficiente de alfa Cronbach entre 0.762 y 0.779 muestran que existe una buena relación entre los ítems indicados en la variable del desarrollo de la competencia léxica oral.

Tabla 1

Coeficientes de Alpha de Crombach

para las dimensiones y competencias

\begin{tabular}{|c|c|}
\hline Dimensiones & Alfa de Cronbach \\
\hline Complejidad (Pre) & 0.7740 \\
\hline Precisión (Pre) & 0.6879 \\
\hline Fluidez (Pre) & 0.7831 \\
\hline Complejidad (Pos) & 0.7187 \\
\hline Precisión (Pos) & 0.7987 \\
\hline Fluidez (Pos) & 0.6758 \\
\hline \multicolumn{2}{|c|}{ Competencias } \\
\hline Léxica Oral (Pre) & 0.7621 \\
\hline Léxica Oral (Pos) & 0.7792 \\
\hline
\end{tabular}

La Tabla 2 muestra las normalidades esperadas de las dimensiones de complejidad, precisión, fluidez y la competencia léxica oral, donde se indican para todas las dimensiones y competencias (Pre) y (Pos) que provienen de una distribución normal a las respuestas observadas con (Val-p >0.100).

Tabla 2

Resultados de la normalidad las dimensiones y competencias

\begin{tabular}{|c|c|c|c|c|c|}
\hline Dimensión & N & Media & Desv.Est. & Rian-Joiner & Val.P \\
\hline Complejidad(Pre) & 23 & 5.217 & 0.9023 & 0.999 & $>0.100$ \\
\hline Complejidad(Pos) & 23 & 13.30 & 1.295 & 1.000 & $>0.100$ \\
\hline Precisión(Pre) & 23 & 5.435 & 1.037 & 0.994 & $>0.100$ \\
\hline Precisión(Pos) & 23 & 12.52 & 0.947 & 0.990 & $>0.100$ \\
\hline Fluidez(Pre) & 23 & 6.626 & 0.606 & 0.999 & $>0.100$ \\
\hline Fluidez(Pos) & 23 & 11.96 & 1.065 & 0.998 & $>0.100$ \\
\hline \multicolumn{7}{|c|}{ Competencias } & \\
\hline Léxica Oral(Pre) & 23 & 16.91 & 1.203 & 0.996 & $>0.100$ \\
\hline Léxica Oral(Pos) & 23 & 37.78 & 1.783 & 0.995 & $>0.100$ \\
\hline
\end{tabular}

El Gráfico 1 muestra la normalidad de las dimensiones (pre y pos) de complejidad, precisión y fluidéz, que parte de una distribución normal, esto debido que los datos están dentro de los limites predichos. 
Gráfico 1

Normalidad de las dimensiones de

Complejidad, precisión y fluidez

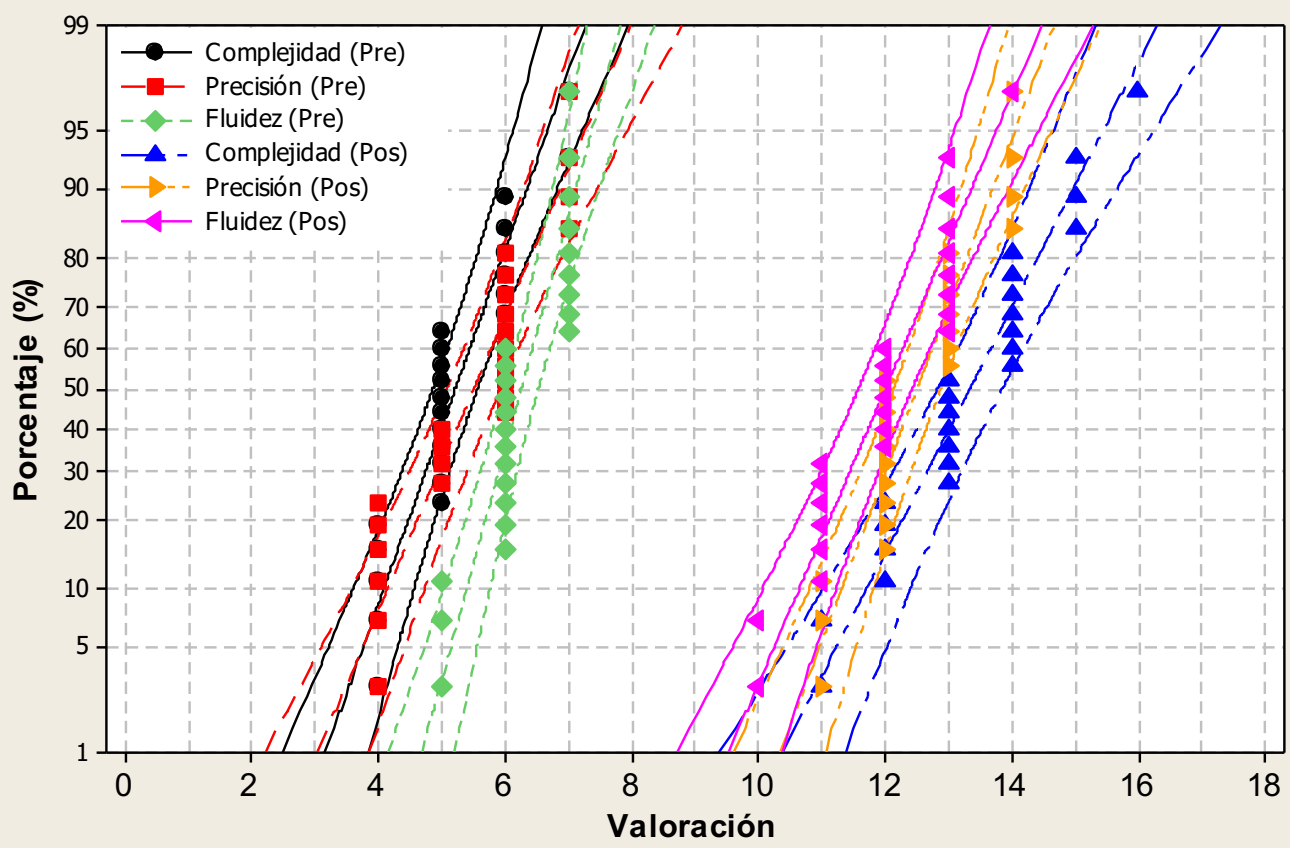

El Gráfico 2 muestra también la normalidad de competencia léxica oral (pre y pos), que parte de una distribución normal, esto debido que los datos están dentro de los limites predichos.

Gráfico 2

Normalidad de competencias léxica oral

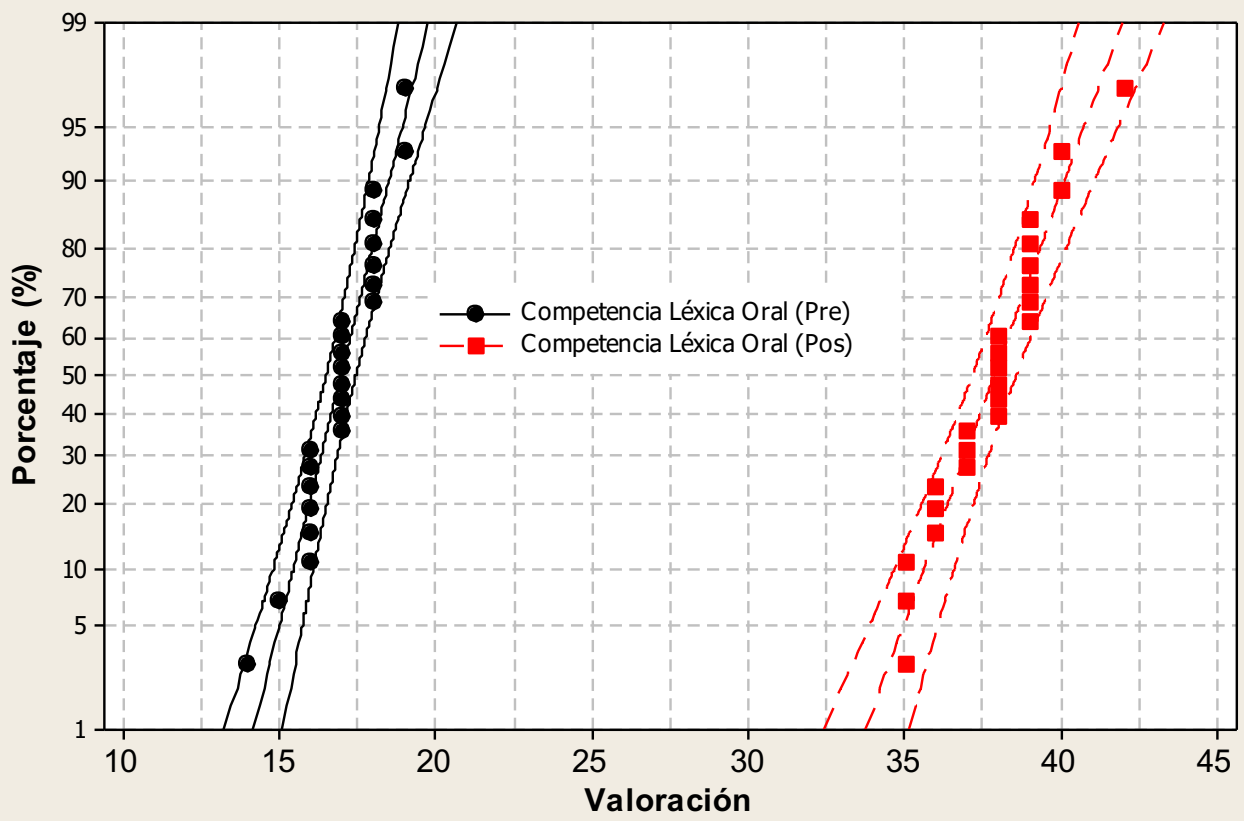

La Tabla 3 muestra las correlaciones de Pearson entre las dimensiones (Pre) y dimensiones (Pos) de complejidad, precisión y fluidez y en los global entre la competencia léxico oral (Pre) y (Pos). La correlación lineal positiva alta 
fue para la dimensiones de precisión (Pre)-Fuidez (Pos) y Precisión (Pre)-Fluidez (Pos) tuvieron coeficientes de correlación de 0.718 y 0.642 respectivamente. La correlación lineal positiva mediana fue para las dimensiones Complejidad (Pre)-Complejidad (Pos), Complejidad (Pre)-Fluidez (Pos), Fluidez (Pre) - Complejidad (Pos) y Fluidez (Pre)-Fluidez (Pos) tuvieron coeficientes de correlación de 0.330, 0.389, 0.366 y 0.388 respectivamente. Y la correlación relativamente negativa neutra fue la dimensión de Fluidez (Pre) - Precisión (Pos). En lo global la correlación lineal de la competencia oral léxica (Pre)-competencia oral léxica (Pos) tuvo un coeficiente de correlación de 0.640 , que existe una correlación positiva relativamente fuerte.

Tabla 3

Coeficientes de correlacion de Pearson para las dimensiones y competencias

\begin{tabular}{|c|c|}
\hline Dimensiones & Correlación de Pearson \\
\hline Complejidad (Pre)- Complejidad (Pos) & 0.330 \\
\hline Complejidad (Pre) - Precisión (Pos) & 0.234 \\
\hline Complejidad (Pre) - Fluidez (Pos) & 0.389 \\
\hline Precisión (Pre) - Precisión (Pos) & 0.234 \\
\hline Precisión (Pre) - Fluidez (Pos) & 0.718 \\
\hline Precisión (Pre) - Complejidad (Pos) & 0.642 \\
\hline Fluidez (Pre) - Complejidad (Pos) & 0.366 \\
\hline Fluidez (Pre) - Precisión (Pos) & -0.009 \\
\hline Fluidez (Pre) - Fluidez (Pos) & 0.388 \\
\hline \multicolumn{2}{|c}{ Competencias } \\
\hline Léxica Oral (Pre) y Léxica Oral (Pos) \\
\hline \multicolumn{2}{|c}{} \\
\hline
\end{tabular}

El Gráfico 3 muestra el comportamiento lineal positivo de las correlaciones de las dimensiones (Pre ) y (Pos), mostrando una pendiente mayor en las dimensiones de precisión (Pre) - Fuidez (Pos) y Precisión (Pre) - Fluidez (Pos), seguido con una pendiente mediana para las dimensiones de Complejidad (Pre)- Complejidad (Pos), Complejidad (Pre) - Fluidez (Pos), Fluidez (Pre) - Complejidad (Pos) y Fluidez (Pre) - Fluidez (Pos).

Gráfico 3

Correlación de las dimensiones de complejidad, precisión y fluidez

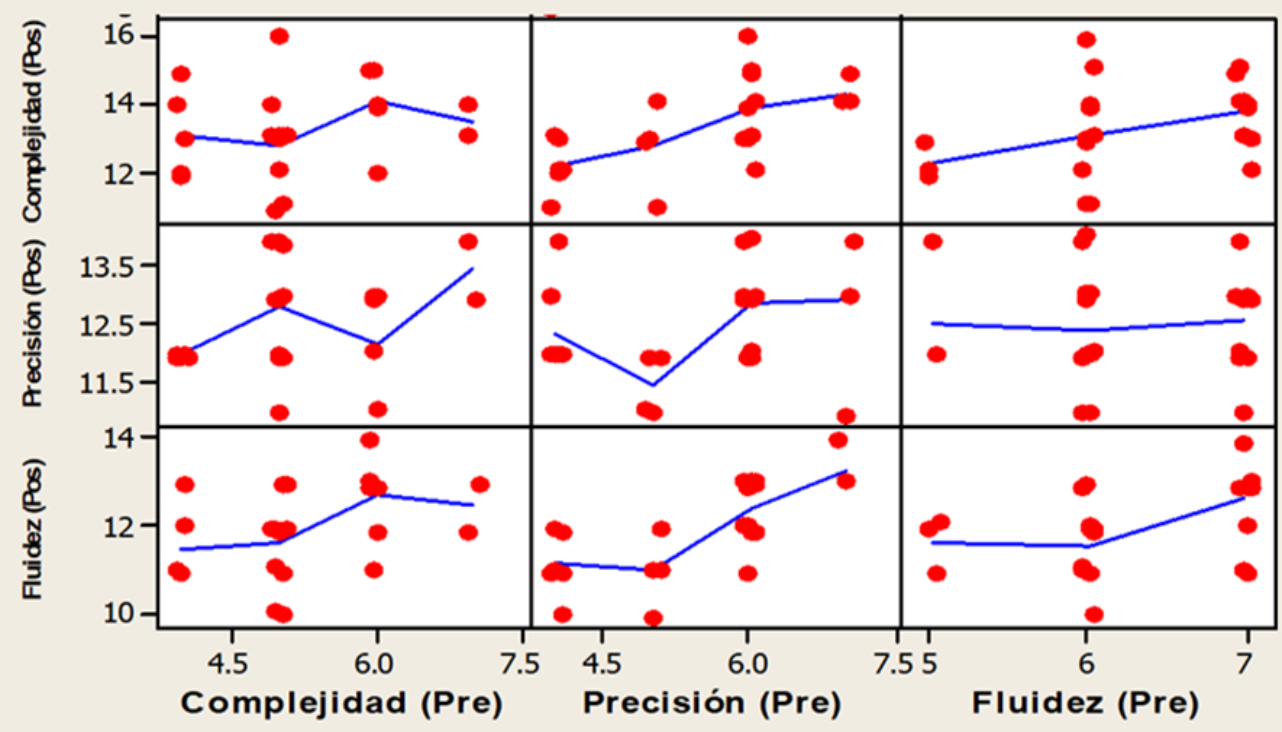


El Grafico 4 muestra el comportamiento lineal de la correlacion global entre la competencia léxica oral (Pre) y léxica oral (Pos), mostrando una fuerte correlación positiva, que a medida que la competencia lexical oral (Pre) es incrementado en su valoración, la competencia léxica oral (Pos) también aumenta en su valoración, eso debido a que existe un buena correlación cuando es empleada la metodología de Enfoque Natural.

\section{Gráfico 4}

Correlación de competencias léxica oral

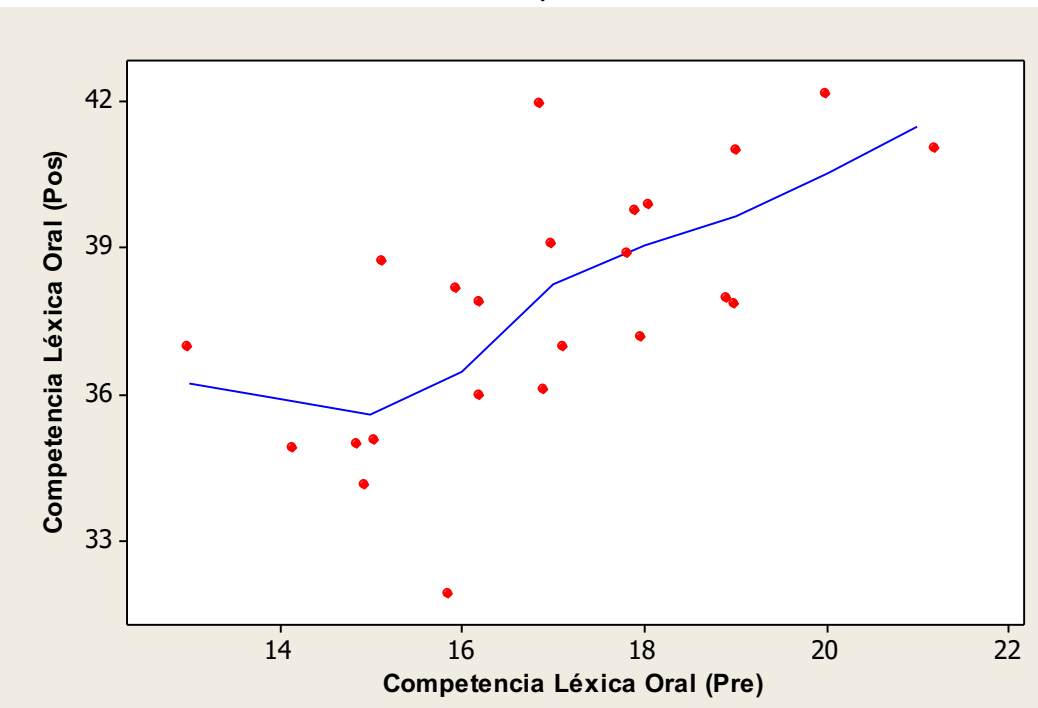

La Tabla 4 muestra los valores de los estimadores-M, donde se observan indicadores de simetría, se puede observar que la media recortada para la dimensión complejidad Pos y Pre fueron de 13.3043 y 5.22 respectivamente, valores relativamente muy parecidos a los estimadores. Para la media recortada de la dimensión precisión Pos y Pre son de 12.5217 y 5.43 respectivamente, valores relativamente parecidos a los estimadores. Igualmente para la media recortada de la dimensión fluidéz Pos y Pre fueron de 11.9565 y 6.26 respectivamente, valores relativamente parecidos a los estimadores.

Finalmente para la media global recortada para competencia Pos y Pre fueron de 37.7826 y 16.91 respectivamente, valores relativamente muy parecidos a los estimadores, señalando que existe estadísticamente una simetría entre las medias y estimadores-M.

Tabla 4

Valores de Estimadores-M para las dimensiones y competencias

\begin{tabular}{|c|c|c|c|c|}
\hline Dimensiones & $\begin{array}{c}\text { Estimador-M de } \\
\text { Huber }^{a}\end{array}$ & $\begin{array}{c}\text { Biponderado de } \\
\text { Tukey }^{b}\end{array}$ & $\begin{array}{c}\text { Estimador-M de } \\
\text { Hampel }^{\mathrm{c}}\end{array}$ & $\begin{array}{l}\text { Onda de } \\
\text { Andrews }\end{array}$ \\
\hline Complejidad (Pos) & 13.265 & 13.270 & 13.301 & 13.271 \\
\hline Complejidad (Pre) & 5.170 & 5.190 & 5.210 & 5.190 \\
\hline Precisión (Pos) & 12.514 & 12.510 & 12.521 & 12.510 \\
\hline Precisión (Pre) & 5.430 & 5.460 & 5.430 & 5.460 \\
\hline Fluidez (Pos) & 11.984 & 11.979 & 11.969 & 11.979 \\
\hline Fluidez (Pre) & 6.320 & 6.430 & 6.380 & 6.610 \\
\hline \multicolumn{5}{|c|}{ Competencias } \\
\hline Léxica oral(Pos) & 37.894 & 37.867 & 37.837 & 37.861 \\
\hline Léxica oral(Pre) & 17.02 & 17.02 & 16.98 & 17.02 \\
\hline
\end{tabular}


La Tabla 5 muestra los resultados estadísticos de la prueba " $\mathrm{T}$ " de Student para muestras pareadas de las dimensiónes de complejidad, precisión, y fluidez, y la variable competencia léxica oral, se observa que el T calculado para las dimensiones de complejidad, precisión y fluidez fue de -29.58, -25.26, -20.55 respectivamente, y para la competencia de lexical oral fue de -55.87 , indicando que todos los valores de " $T$ " calculados son mayores que 2.81 (" $\mathrm{t}$ ", $/ 2=0.025$ ) y también se muestra que el ( $\mathrm{P}$-Val) es $<0.05$ para todas las dimensiones y competencia global, mostrando que existe diferencia significativa al $95 \%$ de intervalo de confianza en la valoración de la dimensión complejidad antes de aplicar el Método Natural Approach (Pre-test) y las valoraciones de la dimensión y competencia, después de aplicar el método Enfoque Natural (Pos-test).

Tabla 5

Muestras pareadas para las

dimensiones y competencias

\begin{tabular}{|c|c|c|c|c|c|}
\hline Dimensión & $\mathbf{N}$ & Media & Desv.Est. & Media E.S. & Pruebas \\
\hline Complejidad(Pre) & 23 & 5.217 & 0.902 & 0.188 & $\mathrm{~T}$ \\
\hline Complejidad(Pos & 23 & 13.304 & 1.295 & 0.270 & -29.58 \\
\hline Diferencia(Pre-Pos) & 23 & -8.087 & 1.311 & 0.273 & Val.P \\
\hline \multicolumn{5}{|c|}{ IC de $95 \%$ para la diferencia media:(-8.654; -7.520$)$} & 0.00001 \\
\hline Precisión(Pre) & 23 & 5.435 & 1.037 & 0.216 & $\mathrm{~T}$ \\
\hline Precisión(Pos) & 23 & 12.522 & 0.947 & 0.198 & -25.26 \\
\hline Diferencia(Pre-Pos) & 23 & -7.087 & 1.345 & 0.281 & Val.P \\
\hline \multicolumn{5}{|c|}{ IC de 95\% para la diferencia media:(-7.669; -6.505) } & 0.00001 \\
\hline Fluidez(Pre) & 23 & 6.261 & 0.689 & 0.144 & $\mathrm{~T}$ \\
\hline Fluidez(Pos & 23 & 11.957 & 1.065 & 0.222 & -20.55 \\
\hline Diferencia(Pre-Pos) & 23 & -5.696 & 1.329 & 0.277 & Val.P \\
\hline \multicolumn{5}{|c|}{ IC de $95 \%$ para la diferencia media: $(-6.270 ;-5.121)$} & 0.00001 \\
\hline \multicolumn{6}{|c|}{ Competencia } \\
\hline Léxica Oral(Pre) & 23 & 16.913 & 1.203 & 0.251 & $\mathrm{~T}$ \\
\hline Léxica Oral(Pos & 23 & 37.783 & 1.783 & 0.372 & -55.87 \\
\hline Diferencia(Pre-Pos) & 23 & -20.87 & 1.792 & 0.374 & Val.P \\
\hline \multicolumn{5}{|c|}{ IC de 95\% para la diferencia media: $(-21.644 ;-20.095)$} & 0.00001 \\
\hline
\end{tabular}

El Gráfico 5 y la Tabla 5 muestran las valoraciones de la media y desviación de la dimensión complejidad pre y pos, los cuales dan un resultado de $5.217 \pm 0.92$ y $13.303 \pm 1.295$ respectivamente, indicando una variación de incremento de 8.09 \pm 1.31 . Para la dimensión Precisión Pre y Precisión Pos fue de $5.435 \pm 1.037$ y $12.522 \pm 0.947$ respectivamente, que señala una variación de incremento de 7.09₫1.35. Por otro lado, en la dimensión Fluidez Pre y Fluidez Pos fue de $6.26 \pm 0.68$ y $11.96 \pm 1.07$ respectivamente, indicando una variación de incremento de $5.70 \pm 1.46$. 
Gráfico 5

Gráfico de cajas de complejidad,

precisión y fluidez

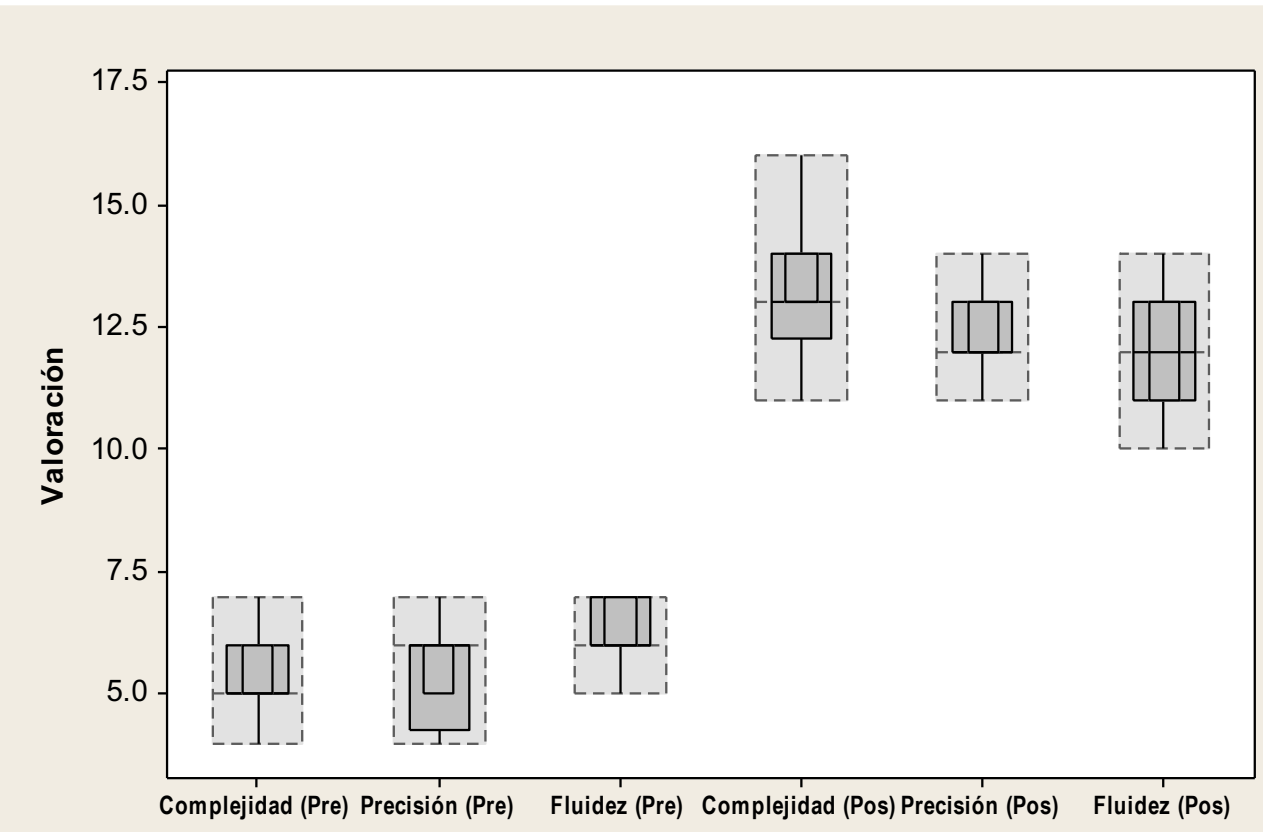

El Gráfico 6 y la Tabla 5 muestran las valoraciones de las media y desviación de la Competencia léxica oral Pre y léxica oral Pos fue de $16.91 \pm 1.78$ y $37.78 \pm 1.78$ respectivamente, indicando una variación de incremento de 20.87 \pm 1.89 . Los incrementos en las valoraciones fueron sustanciales y estadísticamente significativos en la competencia, empleando la metodología de Enfoque Natural.

Gráfico 6

Gráfico de cajas de

competencias oral léxica

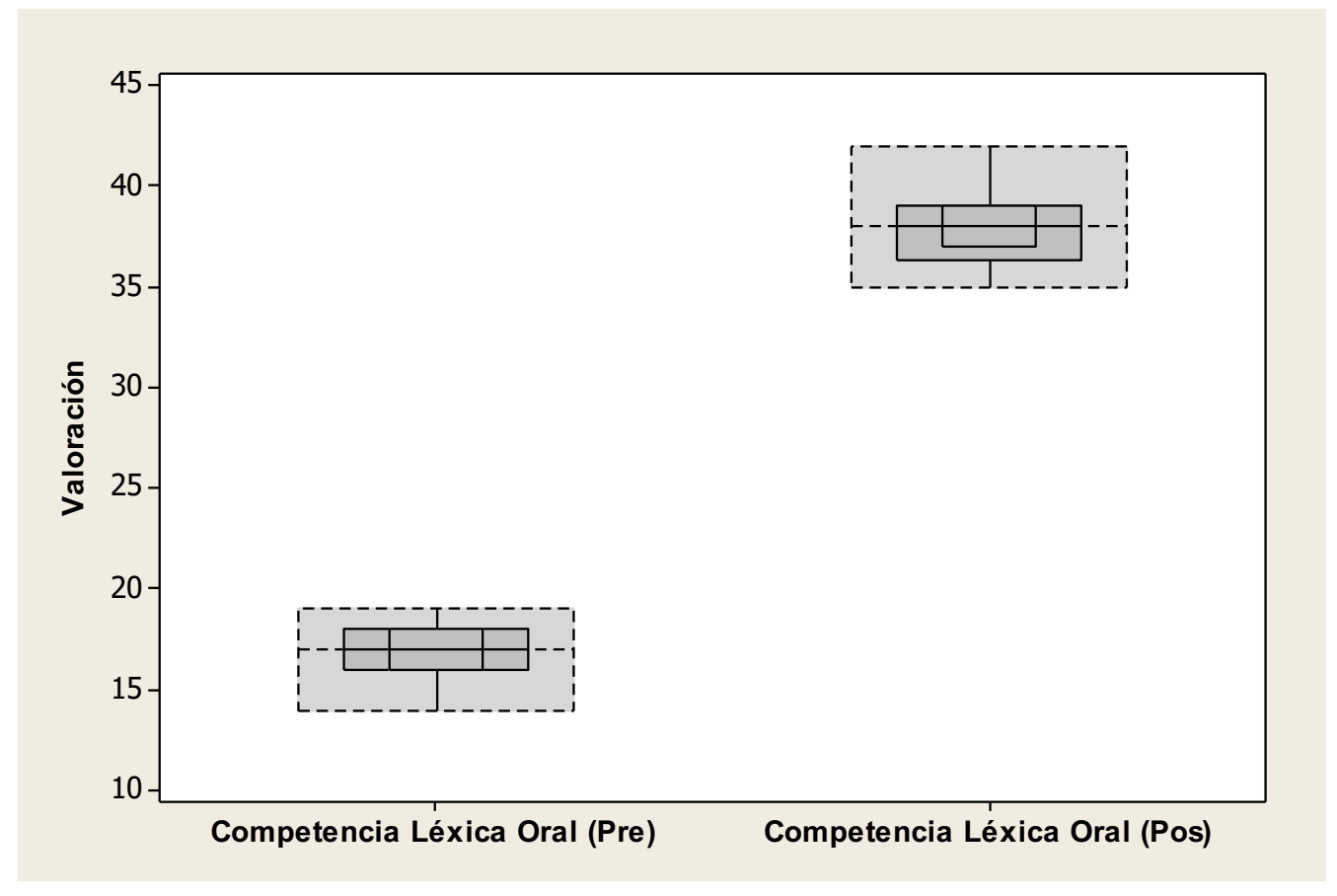


Respecto a los resultados, la Tabla 5 y el Gráfico 5 muestran la efectividad del método Enfoque Natural, con relación a la dimensión complejida del léxico oral, debido a que mostraron una eficiencia de un antes de 5.22 y un después de 13.30. En este sentido Chat et al. (2018) corroboran que al momento de la construcción de un mensaje en inglés, un aprendiz adulto pone mucho énfasis en el significado sobre la forma y focalizándose en la forma de elementos lingüísticos en la complejidad del mensaje, haciendo así un trade off (compensación), el autor realizó un estudio, donde participaron 44 estudiantes de inglés con años y niveles distintos de cada nivel. Participaron 15 estudiantes sumando así un total de 30 estudiantes del nivel A2 y B2; respecto al nivel C1 participaron 14, los cuales fueron seleccionados según los puntajes desarrollados a través del mock exams (simulacro de examen de Cambridge), donde la complejidad se midió en tres niveles: la complejidad global, de frase y por subordinación. Los estudiantes lograron identificar una internalización de nuevos elementos en una lengua extranjera, entendiéndose, así como evidencia de lo necesario de la complejidad del léxico oral. Y López Pérez (2009) menciona que el desarrollo de complejidad sobre los elementos lingüísticos favorecen una mejor comunicación oral (López Pérez, 2009).

Con respecto a los resultados de la dimensión precisión del léxico oral mostrados en la Tabla 5 y Gráfico 5, los resultados fueron estadísticaente significativos donde de un 5.43 antes se pasó a un después de 12.52 mostrando la efectivdad de método Enfoque Natural. En este contexto y según los estudios realizados por Cuitiño, Diaz \& Otárola (2019) han considerado a la precisión del léxico oral como un aspecto fundamental en la expresión oral y así evitar errores en la comprensión de un mensaje de tipo gramatical. Para corroborar el estudio, utilizaron dos test el primero de desempeño oral inicial y uno de desempeño oral final, los resultados del primer test mostraron en sus resultados que un $24 \%$ de estudiantes estuvieron en un nivel excelente, y un $53 \%$ nivel bueno. Respecto al segundo test un $59 \%$ de los estudiantes obtuvieron un nivel excelente, mientras que el $35 \%$ obtuvo el nivel bueno. Cabe resaltar que se usó una rúbrica para ambos test, asimismo, se apoyó con una entrevista semiestructurada consistente en dos preguntas abiertas. Concluyendo así que existió una secuencia didáctica donde se obtuvieron efectos significativos en la mejora de la precisión de léxico oral.

Respecto a los resultados para la fluidez del léxico oral mostrados en la Tabla 5 y Gráfica 5, se obtuvo un resultado significativo de un antes de 6.26 y un después de 11.95 mostrando la eficiencia del método de Enfoque Natural. Coincidiendo con los resultados obtenidos, estudios como el de de De Jong, Groenhout, Schoonen, \& Hulstijn (2015) corroboran la concepción para determinar las medidas de la fluidez del léxico para la competencia oral, donde denota que la personalidad o la forma de hablar de la persona tienen que ver con la fluidez..

Con respecto a los resultados de las variables en estudio de competencia de léxica oral (Tabla 5 y Gráfico 6), antes de la aplicación del método en mención, se obtuvo en el pre test un 16.91, lo que indica un nivel bajo de los estudiantes, sin embargo, luego de la aplicación del método los resultados fueron mayores a un 37.78. Núñez y Del Moral (2010) consideraron que es necesario prestar atención a la competencia léxica ya que, en éstas tres últimas décadas, se han descuidado a través del tiempo los estudios realizados han progresado con lentitud y su enseñanza ha estado dependiendo a las estructuras gramaticales; en ese sentido, los autores corroboran que la teoría y práctica estaban conectados, así hoy en día la adquisición del vocabulario ha tomado fuerza en investigaciones lingüísticas aplicadas. Se menciona que las competencias lingüísticas puede ser analizadas desde niveles superficiales a más profundos haciendo así que el volumen del conocimiento del léxico sea un buen predictor de mejores resultados (Sanhueza C, Ferreira C., \& Sáez C., 2018). Por otro lado el método Enfoque Natural de Krashen y Terrell menciona que la teoría y práctica deben estar conectados, generando así cambios notables en el léxico oral.

Por lo antes mencionado, se puede confirmar que la aplicación del mencionado método en la competencia léxica oral es efectivo, y no solamente el método propuesto sino muchos otros más que se han planteado a lo largo del tiempo. Por eso el maestro debe estar prepajado para que el estudiante, a través de estrategias y aprendizajes 
atractivos, consiga que hasta incluso en zonas rurales, pueda haber un impacto en el aprendizaje del idioma inglés (Ruedas y Wilburn, 2014).

Asímismo, cabe resaltar que el estudio de métodos o su aplicación no ha sido nuevo en la práctica y enseñanza de las lenguas, en este caso el inglés, sino que a lo largo del tiempo siempre se ha buscado su validación teórica y su efectividad (Zanón, 2007). Con base en los resultados obtenidos en la competencia de léxica oral, Barbón et al., (2019) mencionan que la competencia comunicativa en el desempeño comunicativo en lengua inglesa juega un papel determinante en el contexto actual de la colaboración internacional, movilidad académica y desarrollo científico.

\section{Conclusiones}

El método Enfoque Natural fue efectivo, dado que existe una diferencia significativa en los resultados de los niveles obtenidos entre el antes y después, lo que indica efectividad a las dimensiones y competencia léxica oral del inglés de los estudiantes del Instituto de Idiomas de la Universidad Privada.

Con relación a la dimensión complejidad, precisión y fluidez del léxico oral del inglés, tuvieron una diferencia significativa entre los resultados de los niveles obtenidos un antes de $5.22,5.43$ y 6.26 respectivamente, y un después de 13.3, 12.52 y 11.95 respectivamente lo que indica efectividad del método Enfoque Natural respecto a las dimensiones del léxico oral del inglés de los estudiantes.

En la valoración global de competencia, los niveles obtenidos fueron entre un 16.91 antes, y un después de 37.78 con un incremento de 20.87, lo que indica la efectividad del método Enfoque Natural respecto al competencia del léxico oral del inglés de los estudiantes.

Finalmente hay qe destacar que el desempeño comunicativo en lengua inglesa actualmente juega un papel determinante en el contexto mundial de la colaboración internacional, movilidad académica y desarrollo científico.

\section{Referencias bibliográficas}

Barbón, O. G., Morillo, J. R., Nárvaez, M. E., Mejía, E. T., \& González, Y. (2019). La competencia comunicativa en el desempeño comunicativo en Lengua Inglesa de los profesionales de enfermería. Revista Espacios, 40(21), 25-31. Recuperado de: http://www.revistaespacios.com/a19v40n21/a19v40n21p24.pdf

Briseño García, C. (2013). El Léxico del Mundo Animal y el Acceso Léxico Sinònimo en ELE: Estudio Semántico y Didáctico. [Tesis doctoral, Universidad Complutense de Madrid]. Recuperado de: http://eprints.ucm.es/22358/1/T34643.pdf

British Council. (2015). Inglés en el Perú- Un análisis de la politica, las percepciones y los factores de influencia. Educational Intelligence, 64.6 Recuperado de: http://www2.congreso.gob.pe/sicr/cendocbib/con4_uibd.nsf/318A8FA6AC9F382105257F3E00611BB9/\%2 4FILE/Ingl\%C3\%A9s_en_el_Per\%C3\%BA.pdf

Brumfit, C. (1984). Communicative Methodology in Language Teaching: The Roles of Fluency and Accuracy. Cambridge University Press, Cambridge. https://lib.ugent.be/en/catalog/rug01:001447722

Cervilla de Olivieri, M. A. (2009). El caso de la empresa Open English: un emprendimiento global a través de la innovación. Revista $\quad$ Espacios, 15), 19. Recuperado de: http://www.revistaespacios.com/a09v30n01/Textos/CCervilla09-2008.doc 
Chat, J. A., Álvarez, L. G., \& Carrillo, K. S. (2018). Complexity, accuracy and fluency in oral performance of learners with different proficiency levels of English as L2. Revista Signos, 51(96), 3-3. Recuperado de: https://scielo.conicyt.cl/scielo.php?script=sci_arttext\&pid=S0718-09342018000100003

Choez Menoscal, C., Stagg, G., \& Morocho Mazon, M. Y. (2020). Un estudio sobre las estrategias de autorregulación empleadas por aprendices del idioma inglés en una universidad pública del Ecuador. Revista Cubana de Educación Superior. 39(2), 1-15. Recuperado de: http://scielo.sld.cu/scielo.php?script=sci_abstract\&pid=S0257-43142020000200023\&lng=es\&nrm=iso

Cuitiño O. J., Díaz L. C., \& Otárola, J. L. (2019). Promoción de la fluidez y precisión oral en inglés a través del role play. Cuadernos de investigación Educativa, 10(1), 43-62. Recuperado de: http://dx.doi.org/10.18861/cied.2019.10.1.2880

Council of Europe. (2001). Common European Framework of Reference for Languages: learning, teaching, assessment. Council for Cultural Co-operation. Education Committee. Modern Languages Division. Cambridge University Press.

De Jong, N. H., Groenhout, R., Schoonen, R., \& Hulstijn, J. (2015). Second language fluency: Speaking style or proficiency? Correcting measures of second language fluency for first language behavior. Applied Psycholinguistics, 36(2), 223-243. Recuperado de: https://doi.org/10.1017/S0142716413000210

Department of Spanish and Portuguese Studies. (2012). Folwell Hall. University of MinnesotaTwin Cities Minneapolis.

Guacho, Y., Lorena, M., Padilla Padilla, N. M., Padilla Padilla, Y. N., \& Obregon Mayorga, Á. P. (2020). Mejoramiento del Speaking en el idioma inglés con el uso de WhatsApp. Revista Espacios, 41(02), 6. Recuperado de: https://www.revistaespacios.com/a20v41n02/20410206.html

Housen, A., \& Kuiken, F. (2009). Complexity, accuracy, and fluency in second language acquisition. Applied linguistics, 30(4), 461-473. Recuperado de: https://doi.org/10.1093/applin/amp048

Jay Walker, W. (2009). The world's second language. TED Ideas worth spreading: Recuperado de: https://archive.org/details/JayWalker_2009

Julluni Ccahua, N., \& Quispe Samata, Y. (2019). La competencia léxica del idioma inglés en los alumnos del quinto grado de Educación secundaria de la IE Tomasa Ttito Condemayta de a provincia de Acomayo. [Tesis de especialidad, Universidad Nacional San Agustin de Arequipa]. Recuperado de: http://repositorio.unsa.edu.pe/handle/UNSA/8278

Koponen, M. y Riggenbach, H. (2000). Overview: Varying perspectives on fluency, in Riggenbach, H (ed.), Perspectives on fluency, Michigan: University of Michigan Press, 5-24.

Krashen, S. D., y Terrell, T. D. (1983). The natural approach: Language acquisition in the classroom. Researchgate. A Journal of Language Learning, (2), 1-11. Recuperado de: https://doi.org/10.5785/1-2-506

Krashen, S. y Terrell, T. (1988). The Natural Approach. Marylands: Prentice Hall International. Recuperado de: http://www.osea-cite.org/class/SELT_materials/SELT_Reading_Krashen_.pdf

López Pérez, M. (2009). Estrategias y Enfoques metodológicos del uso comunicativo en las lenguas extranjeras: aplicación teórico- práctico del paradigma pragmático. Tejuelo, (07) 57-76. Recuperado de: https://redined.mecd.gob.es/xmlui/handle/11162/28802

Ministerio de Educación. (2020). Diseño Curricular Básico Nacional de la Formación Inicial Docente - Programa de estudios de Educación en Idiomas, especialidad Inglés. Recuperado de: http://www.minedu.gob.pe/superiorpedagogica/producto/dcbn2019-ingles/ 
Morán Flores, G., Arias Chumpitas, A., Fernández Rojas, H., Vilchez de los Rios, A., \& Valladares Alcántara, M. (2010). Instituto Nacional de Estadística e Informática. Recuperado de: https://www.inei.gob.pe/media/MenuRecursivo/censos/cenaun_resultadosdefinitivos-_capitulo_01.pdf

Morante Vallejo, R. (2005). El desarrollo del conocimiento léxico en segundas lenguas. España: LAVEL, S.A.

Mutluoglu, Ö., \& Ceylan, A. (2009). Accuracy and cost comparison of spatial data acquisition methods for the development of geographical information systems. Journal of Geography and Regional Planning, 2(9), 235242. Recuperado de: https://academicjournals.org/journal/JGRP/article-abstract/7F5F41F5789

Nuñez D. P., \& Del Moral BarrigÜete, C. (2010). Competencia léxica y competencia comunicativa: bases para el diseño de programas didácticos en la Educación Escolar. Lenguaje y Textos, 23, 91-97. ISSN: 1133-4470. Recuperado

de: https://digibug.ugr.es/bitstream/handle/10481/24069/Articulo\%20competencia\%20lexica.pdf?sequence= 1

Ramos C. F. (2003). La enseñanza del inglés a estudiantes inmigrantes en Estados Unidos: Un breve resumen de programas y métodos. Revista electrónica de investigación educativa, 5(2), 1-18. Recuperdo de: http://www.scielo.org.mx/pdf/redie/v5n2/v5n2a4.pdf

Romero, E. C. S. (2018). ¿Aproximación Natural o Método Cognitivo? La Enseñanza del Español como Segunda Lengua/Natural Approach OR Cognitive Method? The Teaching of Spanish as a Second Language. Revista Internacional de Ciencias Humanas, 7(1), 1-12. Recuperado de: https://doi.org/10.37467/gkarevhuman.v7.1840

Rueda C. M. C., \& Wilburn D. M. (2014). Enfoques teóricos para la adquisición de una segunda lengua desde el horizonte de la práctica educativa. Perfiles educativos, 36(143), 21-28. Recuperado de: http://www.scielo.org.mx/pdf/peredu/v36n143/v36n143a18.pdf

Sanhueza, C., Ferreira, A., \& Sáez, K. (2018). Desarrollo de la competencia léxica a través de estrategias de aprendizaje de vocabulario en aprendientes de inglés como lengua extranjera. Lexis, 42(2), 273-326. Recuperado de: http://dx.doi.org/10.18800/lexis.201802.002

Skehan, P. (1989) Second language acquisition research and task-based instruction. In J, Willis, \& D, Willis (Eds.), Challenge and change in language teaching, (17-30), Oxford: Heinemann.

Skehan, P. (1996). A framework for the implementation of task-based instruction. Applied linguistics, 17(1), 3862. Recuperado de: https://doi.org/10.1093/applin/17.1.38

Slagter, P. J. (1994). Aproximaciones a cuestiones de adquisición y aprendizaje del español como lengua extranjera o lengua segunda. Atlanta, Georgia: (Vol. 6) Rodopi.

Terrell, T. D. (1985). The natural approach to language teaching: an update. Canadian modern language review, 41(3), 461-479. Recuperado de: https://doi.org/10.3138/cmlr.41.3.461.

Vivanco, V. (2001). Adquisición de Vocabulario en una Segunda Lengua. Estrategias Cognitivas-Lazos Afectivos. Revista de investigación e innovación en la clase de idiomas. 12, 177-187. Recuperado de: https://ebuah.uah.es/xmlui/handle/10017/958-

Zanón, J. (2007). Psicolingüística y didáctica de las lenguas: una aproximación histórica y conceptual. Marco ELE. Revista de Didáctica Español Lengua Extranjera, (5), 1-30. Recuperado de: https://www.redalyc.org/pdf/921/92152376014.pdf 


\section{Anexos}

\section{FICHA DE OBSERVACIÓN DE LA COMPETENCIA LÉXICA DEL INGLÉS}

Nombre del ejecutor:

Nombre del estudiante:

Fecha:

Estudiante de:
Género: (1) Masculino
(2) Femenino

INSTRUCCIONES: Este instrumento tiene como objetivo extraer información acerca del avance del desarrollo de la competencia léxica oral en el idioma Ingles. Esta ficha de observación se aplicará a los estudiantes en dos tiempos antes de ejecutar el método y después (pre- test y posttest)

\section{Grado de logros alcanzados}

\begin{tabular}{|c|c|c|c|}
\hline $\mathbf{1}$ & $\mathbf{2}$ & $\mathbf{3}$ & $\mathbf{4}$ \\
\hline Unsatisfactory (Insatisfactorio) & Low (Bajo) & Medium (Regular) & High (Alto) \\
\hline
\end{tabular}

\begin{tabular}{|c|c|c|c|c|}
\hline Complexity (Complejidad) & 1 & 2 & 3 & 4 \\
\hline $\begin{array}{l}\text { 1. The student develops ideas completely clear and well developed. } \\
\text { El estudiante desarrolla ideas completamente claras y bien construidas. }\end{array}$ & & & & \\
\hline $\begin{array}{l}\text { 2. The student communicates in a logical sequence the message that he/she wants to transmit. } \\
\text { El estudiante comunica en secuencia lógica el mensaje que quiere transmitir. }\end{array}$ & & & & \\
\hline $\begin{array}{l}\text { 3. The student use collocations that cover the class without errors. } \\
\text { El estudiante usa frases (palabras unidas con otras) que cubran la clase sin ningún error. }\end{array}$ & & & & \\
\hline $\begin{array}{l}\text { 4. The student maintains English throughout the conversation without parroting the partner. } \\
\text { El estudiante mantiene el inglés desde el principio hasta el fin de la conversación sin tener que repetir nuevamente a su } \\
\text { compañero. }\end{array}$ & & & & \\
\hline Accuracy (Exactitud) & 1 & 2 & 3 & 4 \\
\hline $\begin{array}{l}\text { 5. The student shows mastery of the grammar presented. Mistakes or errors free, overall (i.e., subject-verb agreement, } \\
\text { noun-adjective agreement, etc.). } \\
\text { El estudiante muestra dominio de la gramática presentada. Libre de errores o fallas (esto es, sujeto-verbo, sustantivo } \\
\text { adjetivos, etc) }\end{array}$ & & & & \\
\hline $\begin{array}{l}\text { 6. The student has the ability to speak without interferences while he/she communicates the message } \\
\text { El estudiante tiene la habilidad de hablar sin interferencias mientras comunica el mensaje. }\end{array}$ & & & & \\
\hline $\begin{array}{l}\text { 7.The student pronounces or makes the sound correctly } \\
\text { El estudiante pronuncia o produce el sonido correctamente. }\end{array}$ & & & & \\
\hline $\begin{array}{l}\text { 8. The student uses the correct and appropriate vocabulary according to the context. } \\
\text { El estudiante usa el vocabulario correcto y apropiado de acuerdo al contexto comunicativo. }\end{array}$ & & & & \\
\hline Fluency (Fluidez) & & & & \\
\hline $\begin{array}{l}\text { 9. The student connects ideas. The student speaks English comfortably. Natural turn-taking. } \\
\text { El estudiante une ideas a través de conectores, logrando así una comunicación sin trabas, tornándolo natural. }\end{array}$ & & & & \\
\hline $\begin{array}{l}\text { 10.The student has the ability to speak easily and smoothly } \\
\text { El estudiante tiene la habilidad de hablar fácilmente y sin (con pocos) problemas }\end{array}$ & & & & \\
\hline $\begin{array}{l}\text { 11. The student must demonstrate fluency when he/she speaks English (foreign language) } \\
\text { El estudiante demuestra fluidez cuando habla en el idioma extranjero }\end{array}$ & & & & \\
\hline $\begin{array}{l}\text { 12. The student speaks clearly and effectively when he or she has a conversation. } \\
\text { El estudiante habla clara y directamente cuando tiene una conversación. }\end{array}$ & & & & \\
\hline
\end{tabular}

Evaluación oral en inglés, basada y adaptada en: Department of Spanish and Portuguese Studies, (2012).

\section{SESIONES DE CLASES SEGÚN EL MÉTODO NATURAL APPROACH \\ LESSON PLAN 1}

\section{General Information}

Number of students: 23 students

Area: Basic II

Lesson plan topic: Meeting people

Class Period Time: 135 Minutes

Performance/ Task-based Objectives: Students will be able verbally introduce themselves in target language. Students will be able to choral repeat with teacher.

\section{THE NATURAL APPROACH IN THE CLASSROOM}

The Natural Approach is designed to develop basic communication skills. The development stages are: (1) Comprehension (preproduction), (2) Early Production, (3) Speech Emergence and (4) Intermediate Fluency. This approach to teaching language has been proven to be particularly effective with limited English proficient students.

\section{STAGE I COMPREHENSION}


In order to maximize opportunities for comprehension experiences. Natural Approach instructors (1) create activities designed to teach students to recognize the meaning in words used in meaningful contexts, and (2) teach students to guess at the meaning of phrases without knowing all of the words and structures of the sentences. a) The teacher ALWAYS USE VISUAL AIDS (pictures, realia, gestures). b) The teacher helps to MODIFY YOUR SPEECH to aid comprehension, speak more slowly, emphasize key words, simplify vocabulary and grammar, use related ideas, do not talk out of context. c) The teacher DO NOT FORCE PRODUCTION. Students will use English when they are ready. They sometimes experience a "silent period" which can last days or weeks.d) FOCUS ATTENTION ON KEY VOCABULARY.

Teacher Activities in the Comprehension Stage.

a) Total Physical Response (TPR). The teacher gives commands to which the students react with their bodies as well as their brains. b) Supplying meaningful input based on items in the classroom or brought to class. This is according to the topic:

What is your name? Where are you from? Wow are you today? c) Supplying meaningful input based on pictures.

Student Responses in the Comprehension Stage.

a. An action (TPR). b. The name of a fellow student (from b., c. above). c. Gestures d. Students say yes/no in English. e. Students point to an item or picture. f. Children do not initially make many attempts to communicate using words, rather they indicate their comprehension nonverbally. Sesiones de aplicación basado en el Departamento de idiomas de la universidad de California (Terrell, 1990).

\section{LESSON PLAN 02}

General Information

Number of students: 23 students

Area: Basic II

Lesson plan topic: What kind of person are you? Describing people.

Class Period Time: 135 Minutes

Performance/ Task-based Objectives:

Students will be able verbally describe themselves in target language.

Students will be able to choral repeat with teacher and learn the vocabulary.

THE NATURAL APPROACH IN THE CLASSROOM

The Natural Approach is designed to develop basic communication skills. The development stages are: (1) Comprehension (preproduction), (2) Early Production, (3) Speech Emergence and (4) Intermediate Fluency. This approach to teaching language has been proven to be particularly effective with limited English proficient students.

\section{STAGE I COMPREHENSION}

In order to maximize opportunities for comprehension experiences. Natural Approach instructors (1) create activities designed to teach students to recognize the meaning in words used in meaningful contexts, and (2) teach students to guess at the meaning of phrases without knowing all of the words and structures of the sentences.

a) The teacher ALWAYS USE VISUAL AIDS (pictures, realia, gestures). b) The teacher helps to MODIFY YOUR SPEECH to aid comprehension, speak more slowly, emphasize key words, simplify vocabulary and grammar, use related ideas, do not talk out of context. c) The teacher DO NOT FORCE PRODUCTION. Students will use English when they are ready. They sometimes experience a "silent period" which can last days or weeks. d) FOCUS ATTENTION ON KEY VOCABULARY.

Teacher Activities in the Comprehension Stage.

a) Total Physical Response (TPR). The teacher gives commands to which the students react with their bodies as well as their brains. b) Supplying meaningful input based on items in the classroom or brought to class (This is according to the topic: What kind of person are you? Can you describe a person with one adjective? Who has the brown hair? Who is wearing a red jacket). c) Supplying meaningful input based on pictures.

Student Responses in the Comprehension Stage.

a) An action (TPR). b. The name of a fellow student (from b., c. above). c) Gestures, d) Students say yes/no in English. e) Students point to an item or picture. f) Children do not initially make many attempts to communicate using words, rather they indicate their comprehension nonverbally. Sesiones de aplicación basado en el Departamento de idiomas de la universidad de California (Terrel, 1990).

\section{LESSON PLAN 03}

General Information

Number of students: 23 students

Area: Basic II

Lesson plan topic: Which stores do you like to shop at?

Class Period Time: 135 Minutes

Performance/ Task-based Objectives:

Students will be able to describe verbally the stores where they like to shop and, the size and clothes.

Students will be able to choral repeat with teacher and learn the vocabulary.

\section{THE NATURAL APPROACH IN THE CLASSROOM}

The Natural Approach is designed to develop basic communication skills. The development stages are: (1) Comprehension (preproduction), (2) Early Production, (3) Speech Emergence and (4) Intermediate Fluency. This approach to teaching language has been proven to be particularly effective with limited English proficient students.

\section{Stage 2 EARLY SPEECH}

In non-threatening environments, students move voluntarily into Stage 2. Stage 2 begins when students begin using English words to give: a) yes/no answers, b) one-word answers, c) lists of words, d. two word strings and short phrases

The following are instructor question techniques to encourage the transition from Stage 1 to Stage 2., a) Yes/no questions (Do you like to buy in the stores?), b) Choice questions (Is this a orange or an limon?), c) Questions which can be answered with a single word (What does the children have in their hands? Is it a book or some fruit?. Where can you buy When is your favorite day that you can buy? Who is a vegetarian in your 
family?. d) General questions which encourage lists of words. What things do we see on the table now? e) Open sentence with pause for student response.

Jorge is eating an apple now, but Ramon is eating a

During the Early Speech Stage, the instructor must give a meaningful and understandable input which will encourage the transition to Stage 3. Therefore all student responses should be expanded if possible. Here is a sample exchange between the teacher and the class:

Teacher: What do we see in these pictures?

Class: fruits. Teacher: Yes, there are some fruits in these pictures. Is there a banana?. Class: Yes. Teacher: Yes, there is. There is a watermelon and grapes. Where is the watermelon?. Class: In the store. Teacher: Yes that's right. The watermelon is in the store. Is somebody eating?. Class: Yes. Other sorts of activities is used in Early Speech Stage: a) open dialogues, b) guided interviews, c) open-ended sentences, d) charts, tables, graphs, e) newspaper ads.

Sesiones de aplicación basado en el Departamento de idiomas de la universidad de California (Terrel, 1990).

\section{LESSON PLAN 04}

General Information

Number of students: 23 students

Area: Basic II

Lesson plan topic: TV Shows

Class Period Time: 135 Minutes

Performance/ Task-based Objectives:

Students will be able to recognize every TV programs and describe the name of them.

Students will be able to choral repeat with teacher and learn the vocabulary.

THE NATURAL APPROACH IN THE CLASSROOM

The Natural Approach is designed to develop basic communication skills. The development stages are: (1) Comprehension (preproduction), (2) Early Production, (3) Speech Emergence and (4) Intermediate Fluency. This approach to teaching language has been proven to be particularly effective with limited English proficient students.

\section{Stage 2 EARLY SPEECH}

- In non-threatening environments, students move voluntarily into Stage 2. Stage 2 begins when students begin using English words to give:

a) yes/no answers, b) one-word answers, c) lists of words, d. two word strings and short phrases. The following are instructor question techniques to encourage the transition from Stage 1 to Stage 2: a) Yes/no questions (Do you like the movies?), b. Choice questions (Is this a video a movie or a opera?), c) Questions which can be answered with a single word. What's is a movie? What's a soap opera? Did you participate in a reality show? What do you do in a game show? d. General questions which encourage lists of words. What video do we watch in the video now?

e) Open sentence with pause for student response. Jorge likes stories, but Ramon likes

During the Early Speech Stage, the instructor must give a meaningful and understandable input which will encourage the transition to Stage 3. Therefore all student responses should be expanded if possible. Here is a sample exchange between the teacher and the class:

Teacher: What do we see in these videos?

Class: movies. Teacher: Yes, there are some movies. Exist some differences between videos and soap opera? Class: Yes. Teacher: Yes, of course. What is the most important part in a movie?. What is the man doing? Class: He is giving something to the poor. Teacher: Yes that's right. The man is giving some food to the poor and they are eating? Class: Yes.

Other sorts of activities is used in Early Speech Stage: a) open dialogues, b) guided interviews, c) open-ended sentences, d) charts, tables, graphs, e) newspaper ads

Sesiones de aplicación basado en el Departamento de idiomas de la universidad de California (Terrell, 1990).

\section{LESSON PLAN 05}

General Information

Number of students: 23 students

Area: Basic II

Lesson plan topic: Technology now and the future

Class Period Time: 135 Minutes

Performance/ Task-based Objectives:

Students give opinion about the world and learn new vocabulary. Students will be able to choral repeat with teacher and learn the vocabulary. THE NATURAL APPROACH IN THE CLASSROOM

The Natural Approach is designed to develop basic communication skills. The development stages are: (1) Comprehension (preproduction), (2) Early Production, (3) Speech Emergence and (4) Intermediate Fluency. This approach to teaching language has been proven to be particularly effective with limited English proficient students.

\section{Stage 3 SPEECH EMERGENCE}

In the Speech Emergence Stage, speech production will normally improve in both quantity and quality. The sentences that the students produce become longer, more complex and they use a wider range of vocabulary. Finally, the number of errors will slowly decrease.

Students need to be given the opportunity to use oral and written language whenever possible. When

they reach the stage in which speech is emerging beyond the two-word stage, there are many sorts of activities which will foster more comprehension and speech. Some suggestions are: a) preference ranking, b) games of all sorts, c) problem-solving using charts, tables graphs, maps, d) advertisements and signs, e) group discussion, f) skits (finger plays, flannel boards, puppets), g) music, radio, television, film strips, slides, h) writing exercises (especially Language Experience Approach), i) reading, j) culture.

In general, we may classify language acquisition activities as those in which the focus is on the message, i.e., meaning. These may be of four types: a) content (culture, subject matter, new information, reading), b) affective-humanistic (student's own ideas, opinions, experiences), c) games (focus on using language to participate in the game), d) problem-solving (focus on using language to locate information) 
Methods in Teaching Through Total Physical Response

\section{Orientation}

To introduce and motivate the class: Have a translator briefly explain the theory behind the method, Show a documentary film of students learning through TPR, or, Say commands rapidly in English and announce in the student's language that by the end of the class everyone will understand everything that you just said.

\section{Preparation}

Before the teacher begins each lesson plan: Have a detailed outline or script of the elements that you will teach, the various combinations and recombinations of elements, zany commands, and a strategy for varying from individuals to small and large group movement. Get props together and have them handy; Arrange the class so that there is a large space for the action and so that everyone can see (possibly a semi-circle).

\section{Classroom Procedure}

A. The Method (taken from Teaching English Through Action )

a) Demonstration - the students listen and respond to commands modeled by the teacher. (1) Teacher commands and models with the entire group. (2) I Teacher commands and models with 2-3 or 4-6 students. (3) Teacher commands and models with 1 student. b) Group responds to commands without teacher. c) Group of 3-5 students responds to commands without teacher. d) Individual student responds to commands without teacher. e) Instructor recombines old and new commands and models with the group: (1) Group responds to recombined commands without teacher. (2) 2-3 students respond to recombined commands: Without teacher modeling and Without teacher.

B. Progression of Commands - The steps in the development of a lesson plan look something like this...

a) Simple actions ("walk", "jump"), b) Simple actions involving objects and locations ("walk to the door"), c) Recombinations of actions and objects ("walk to the chair", "touch the chair"), d) Recombinations of actions and objects involving transferring meaning to a new situation ("shake your head", "shake my hand"), e) Chains of actions leading into an activity sequence ("Take the can", "Open the can", "Pour the water" .... "Drink the lemonade").

C. Some pointers: a) Model 'clean' responses to commands so that students will not pick up extraneous gestures that are false to the meaning of the command. (For example, don't swivel your head and then turn around with the command "turn".), b) Novel commands (new combinations of elements already mastered keep interest high and enhance self-confidence as students realize they have understood something never quite heard before.), c) Introduce new vocabulary 3 items at a time and proceed only after students are responding confidently., d) If students do not grasp a new item after a few trials, drop it until a future time. (For example, students may not be able to transfer from "point to the corner of your eye" to "walk to the corner of the room".), e) When commanding individuals, call on confident students. Sometimes invite volunteers by saying "one student". A shy student may jump up and carry out a command because she or he was the first to understand it, f. Keep varying who you call on by asking all the women, all the students on the right side, near the window, in row one, from Cuba. This keeps the students alert, never knowing who you will call on next, g. Keep changing the order of the commands to increase listening attention.

D. The Expressive Stage (Speaking): 1) After about 10 hours of TPR the students will begin to reveal a readiness to speak by mouthing or mumbling your commands out loud. At this point you can: a) Invite the students to command the teacher, other students, or the whole group: b) Ask questions that involve yes or not answers. (Look at the clock." "Is it 5 o'clock?"); c) Progress to questions involving one word answers ("Go home." "Where's he going?"), 2) Students will begin to lengthen their answers as they hear and assimilate more. They will improve word order and pronunciation through closer and closer approximation of what they hear. 3) As students become more proficient, the instructor can add substitution drills, transformation drills, dialogues, and conversations.

E. The Expressive Stage (Written): 1) The instructor can give out study papers after a few lessons with the words used in class, demonstrating and saying each of the words. The students use the papers as they wish. This is good for those students who wish to have it "down". 2) For illiterate students or very basic beginners, numbers and simple words can be manipulated on cards ("put number 5 in front of number 2"). Commands can also include blackboard tasks ("Circle the date" or "Write your name next to number 1."). 3) Reading and Writing lessons can increase in complexity as the students progress.

Sesiones de aplicación basado en el Departamento de idiomas de la universidad de California (Terrell, 1990).

\section{LESSON PLAN 06}

General Information

Number of students: 23 students

Area: Basic II

Lesson plan topic: Cooking

Class Period Time: 135 Minutes

Performance/ Task-based Objectives: Students give opinions about the foods and learn new vocabulary. Students will be able to choral repeat with teacher and learn the vocabulary.

\section{THE NATURAL APPROACH IN THE CLASSROOM}

The Natural Approach is designed to develop basic communication skills. The development stages are: (1) Comprehension (preproduction), (2) Early Production, (3) Speech Emergence and (4) Intermediate Fluency. This approach to teaching language has been proven to be particularly effective with limited English proficient students.

\section{Stage 3 SPEECH EMERGENCE}

In the Speech Emergence Stage, speech production will normally improve in both quantity and quality. The sentences that the students produce become longer, more complex and they use a wider range of vocabulary. Finally, the number of errors will slowly decrease.

Students need to be given the opportunity to use oral and written language whenever possible. When they reach the stage in which speech is emerging beyond the two-word stage, there are many sorts of activities which will foster more comprehension and speech. Some suggestions are:

A. Preference ranking, b. games of all sorts, c. problem-solving using charts, tables graphs, maps, d. advertisements and signs, e. group discussion, f. skits (finger plays, flannel boards, puppets), g. music, radio, television, film strips, slides, h. writing exercises (especially Language Experience Approach), i. reading, j. culture 
B. In general, we may classify language acquisition activities as those in which the focus is on the message, i.e., meaning. These may be of four types:

a. content (culture, subject matter, new information, reading), b. affective-humanistic (student's own ideas, opinions, experiences), c. games (focus on using language to participate in the game), $d$. problem-solving (focus on using language to locate information)

Sesiones de aplicación basado en el Departamento de idiomas de la universidad de California (Terrell, 1990).

Esta obra está bajo una Licencia Creative Commons

Attribución-NoCommercial 4.0 International

(cc) EY-NC 